\title{
The logistic population model with slowly varying carrying capacity
}

\author{
J. J. Shepherd* I. Stojkov*
}

(Received 14 November 2005; revised 30 November 2006)

\begin{abstract}
Many single-species differential equation population models feature a carrying capacity - the limiting population supportable by the environment. For constant carrying capacities an exact solution may often be found, representing the evolving population in time. However, for time varying carrying capacities, exact solution is rarely possible, and numerical techniques must be used. We demonstrate that when the carrying capacity varies slowly with time, a multiple time scale analysis leads to approximate closed form solutions that, apart from being explicit, are comparable to numerically generated ones and which are valid for a range of parameter values.
\end{abstract}

${ }^{*}$ School of Mathematical \& Geospatial Sciences, RMIT University, PO Box 2476V, Melbourne, Victoria 3001, Australia. mailto: jshep@rmit.edu.au

See http://anziamj.austms.org.au/V47EMAC2005/Shepherd for this article, (C) Austral. Mathematical Soc. 2007. Published January 4, 2007. ISSN 1446-8735 


\section{Contents}

1 Introduction

C493

2 The slowly-varying logistic equation

C495

3 Perturbation analysis

C496

4 The power law logistic equation

C499

5 Discussion

C500

References

C505

\section{Introduction}

The modelling of the evolution of populations using differential equations is a well established practice that has a long history. While it is true that populations of individuals change in discrete steps, their representation by continuous variables enables the use of techniques that have been shown to yield useful results in many areas of application. If spatial effects such as diffusion and dispersion are neglected, the mathematical model of a single species population then reduces to an initial-value problem involving a single (usually nonlinear) ordinary differential equation in one variable $[6$, e.g.]. Although such an apparently gross simplification may be criticised, such models still find application in the study of such disparate phenomena as the evolution of fish school populations and the spread of a single innovation or phenomenon $[4,5]$.

Many of these single species models feature a carrying capacity, a term used to denote the limiting population supportable by the environment. In the simplest instance, this is a constant; and in such cases, considerable 
progress may be made in the analysis of the underlying differential equation. Often, this may be solved exactly, to yield an explicit expression for the evolving population. In other cases, indirect methods may be used to deduce the overall population behaviour. However, when the carrying capacity varies with time, it is not usually possible to solve the differential equation exactlyalthough some exact results do exist [1]. Thus, approximate methods must be resorted to; in particular, numerical techniques. These have the disadvantage of applying only to particular values of any parameters, not a range.

In a large class of phenomena, the carrying capacity varies slowly, relative to the changing population. This may arise from slow changes in the population species itself, or in the background environment, or a combination of both. In such cases, the carrying capacity may be represented as a (positive valued) function $K(\varepsilon t)$, where $t$ is time, and $\varepsilon$ is a small, positive parameter. Thus, to produce a "normal size" change in the value of $K, t$ must change by an amount of "size" $1 / \varepsilon$; that is, $K(\varepsilon t)$ is slowly varying in time $t$.

We demonstrate a technique that constructs an approximation to the population, valid for all times, when the carrying capacity is slowly varying as described above. This method belongs to a class of related methods, termed multi-timing methods, which are well established in physics and engineering $[7,3$, e.g.]. Such methods exploit the disparate time variation between components of a system, to produce an algorithm capable of generating an approximate solution to problems involving the system. In the present case, the disparity is between the variation of population and the carrying capacity. Since the details are heavily dependent on the structure of the underlying differential equation, we confine our attention to two particular but useful population models - the logistic and power law logistic equations. However, bear in mind that the method is readily applicable to a wide range of such models displaying slowly varying carrying capacity. Where possible, we compare the results provided by the multi-timing method with exact solutions, when they exist; or, failing that, with the results of numerical calculations 


\section{The slowly-varying logistic equation}

If, as discussed above, the carrying capacity is characterised by a function $K(\varepsilon t)$, where $\varepsilon$ is a small, positive parameter, the logistic, initial value problem for the population $P(t, \varepsilon)$ at all times $t \geq 0$, with starting population $P_{0}$ is the differential equation

$$
\frac{d P(t, \varepsilon)}{d t}=r P(t, \varepsilon)\left[1-\frac{P(t, \varepsilon)}{K(\varepsilon t)}\right], \quad P(0, \varepsilon)=P_{0},
$$

where $r$ is a positive constant.

In principle, given $r$ and the function $K(\varepsilon t)$, solution of (1) yields $P(t, \varepsilon)$. However, in practice, for arbitrary functions $K(\varepsilon t)$, exact solution of (1) is not possible, and numerical solutions must be resorted to. These have the disadvantage of being available only for specific values of $r, \varepsilon$ and $P_{0}$, so that an understanding of the behavior of $P(t, \varepsilon)$ over a range of values of these parameters may only be obtained as a result of numerous recalculations. In this investigation, we propose a different approach that will yield an approximation to $P(t, \varepsilon)$ valid for small (but otherwise arbitrary) values of $\varepsilon$ and arbitrary assigned functions $K(\varepsilon t)$. We begin by noting that solutions $P(t, \varepsilon)$ of (1) may be viewed as depending on "ordinary" time, $t_{0}=t$, and "slow" time, $t_{1}=\varepsilon t$, and propose that $P(t, \varepsilon)$ is expressible as a function $p$ of $t_{0}, t_{1}$ (and $\varepsilon$ ); that is

$$
P(t, \varepsilon) \equiv p\left(t_{0}, t_{1}, \varepsilon\right)
$$

From (2), we deduce that the derivative of $P$ with respect to time, $t$, transforms according to the chain rule

$$
\frac{d P}{d t} \equiv \frac{\partial p}{\partial t_{0}}+\varepsilon \frac{\partial p}{\partial t_{1}}
$$

If (3) is substituted into (1), we obtain (1) in the form

$$
\frac{\partial p}{\partial t_{0}}+\varepsilon \frac{\partial p}{\partial t_{1}}=r p\left[1-\frac{p}{K\left(t_{1}\right)}\right], \quad p(0,0, \varepsilon)=P_{0} .
$$


Equation (4) is the multiscaled form of the problem (1). The two derivatives on the left hand side show explicitly the variation of $p$ with respect to $t_{0}$ and the (much lesser) variation with respect to "slow time", $t_{1}$. Now, the ordinary differential equation in (1) has been converted to a partial differential equation - a considerable increase in complexity.

\section{Perturbation analysis}

The conversion of the initial value problem (1) to (4) above reformulates the differential equation in a way that displays the small parameter $\varepsilon$ explicitly, and leads to the possibility of solving (4) approximately using a perturbation expansion. To this end, we propose that $p$, the (exact) solution of (4) be expressible as a perturbation expansion

$$
p\left(t_{0}, t_{1}, \varepsilon\right)=p_{0}\left(t_{0}, t_{1}\right)+\varepsilon p_{1}\left(t_{0}, t_{1}\right)+\varepsilon^{2} p_{2}\left(t_{0}, t_{1}\right)+\cdots ;
$$

where the coefficient functions $p_{i}\left(t_{0}, t_{1}\right)$ are to be determined. If we now substitute the expansion (5) into (4), and equate like powers of $\varepsilon$, we obtain the differential equations

$$
\begin{aligned}
& \frac{\partial p_{0}}{\partial t_{0}}=r p_{0}\left[1-\frac{p_{0}}{K\left(t_{1}\right)}\right] \\
& \frac{\partial p_{1}}{\partial t_{0}}-r p_{1}\left[1-\frac{2 p_{0}}{K\left(t_{1}\right)}\right]=-\frac{\partial p_{0}}{\partial t_{1}}
\end{aligned}
$$

for $p_{0}, p_{1}$ respectively; with subsequent analogous equations for the later coefficient functions.

Solving the partial differential equation (6) for $p_{0}$ gives

$$
p_{0}\left(t_{0}, t_{1}\right)=\frac{K\left(t_{1}\right)}{1+A\left(t_{1}\right) e^{-r t_{0}}},
$$


where $A\left(t_{1}\right)$ is an arbitrary function of $t_{1}$ alone. With $p_{0}$ given by (8), (7) becomes

$$
\begin{aligned}
& \frac{\partial p_{1}}{\partial t_{0}}-\left(1-\frac{2}{1+A\left(t_{1}\right) e^{-r t_{0}}}\right) r p_{1} \\
= & \frac{K\left(t_{1}\right) A^{\prime}\left(t_{1}\right) e^{-r t_{0}}}{\left(1+A\left(t_{1}\right) e^{-r t_{0}}\right)^{2}}-\frac{K^{\prime}\left(t_{1}\right)}{1+A\left(t_{1}\right) e^{-r t_{0}}} .
\end{aligned}
$$

A particular solution for (9) is

$$
p_{1}=\frac{\left(K\left(t_{1}\right) A^{\prime}\left(t_{1}\right)-K^{\prime}\left(t_{1}\right) A\left(t_{1}\right)\right) t_{0} e^{-r t_{0}}}{\left(1+A\left(t_{1}\right) e^{-r t_{0}}\right)^{2}}-\frac{K^{\prime}\left(t_{1}\right)}{r\left(1+A\left(t_{1}\right) e^{-r t_{0}}\right)^{2}} .
$$

The expression (10) contains the term $t_{0} e^{-r t_{0}}$, which does decay as $t_{0} \rightarrow \infty$, but not as rapidly as $e^{-r t_{0}}$, the decay rate of $p_{0}$. Thus, to avoid the possibility of the second term of (5) becoming larger than the first for some $t_{0}$, we choose the function $A\left(t_{1}\right)$ to satisfy

$$
K\left(t_{1}\right) A^{\prime}\left(t_{1}\right)-K^{\prime}\left(t_{1}\right) A\left(t_{1}\right)=0,
$$

giving

$$
A\left(t_{1}\right)=c K\left(t_{1}\right)
$$

where $c$ is an arbitrary constant, so that (10) becomes

$$
p_{1}=-\frac{K^{\prime}\left(t_{1}\right)}{r\left(1+c K\left(t_{1}\right) e^{-r t_{0}}\right)^{2}} .
$$

We choose $p_{1}$ to be this particular solution, since inclusion of a complementary function in $p_{1}$ would ultimately invoke a second arbitrary constant. (The problem (1) is first order, so its solution should involve only one constant, although this constant may depend on $\varepsilon$.) With $p_{0}$ and $p_{1}$ determined as above, the two term expansion for $P(t, \varepsilon)$ is, in the original variables,

$$
P(t, \varepsilon)=\frac{K(\varepsilon t)}{1+c K(\varepsilon t) e^{-r t}}-\varepsilon \frac{K^{\prime}(\varepsilon t)}{r\left(1+c K(\varepsilon t) e^{-r t}\right)^{2}}+O\left(\varepsilon^{2}\right) .
$$


Note that (11) depends on $t, \varepsilon t$, and the arbitrary constant $c$, which may be evaluated by application of the initial condition in (1). Application of this to (11) gives

$$
r P(0)(1+c K(0))^{2}=r K(0)(1+c K(0))-\varepsilon K^{\prime}(0)+O\left(\varepsilon^{2}\right) .
$$

Equation (12) determines $c$ (which depends on $\varepsilon$ ). Proposing an expansion for $c$ of the form $c=c_{0}+\varepsilon c_{1}+\varepsilon^{2} c_{2}+\cdots$, where $c_{0}$ and $c_{1}$ are independent of $\varepsilon$, and substituting into (12) gives, on equating like powers of $\varepsilon$, equations determining $c_{0}, c_{1}, \cdots$. Carrying out the necessary algebraic manipulations, we obtain

$$
c_{0}=\frac{1}{P_{0}}-\frac{1}{K(0)}, \quad c_{1}=-\frac{K^{\prime}(0)}{r K(0)^{2}} .
$$

Replacing $c$ in (11) with $c_{0}+\varepsilon c_{1}$, when $c_{0}$ and $c_{1}$ are given by the values above, and expanding the resulting expression for small $\varepsilon$ gives the final expansion for $P(t, \varepsilon)$ as

$$
\begin{aligned}
P(t, \varepsilon)= & \frac{K(\varepsilon t)}{1+\left(\frac{1}{P_{0}}-\frac{1}{K(0)}\right) K(\varepsilon t) e^{-r t}} \\
& -\varepsilon \frac{K^{\prime}(\varepsilon t) K(0)^{2}-K^{\prime}(0) K(\varepsilon t)^{2} e^{-r t}}{r K(0)^{2}\left(1+\left(\frac{1}{P(0)}-\frac{1}{K(0)}\right) K(\varepsilon t) e^{-r t}\right)^{2}}+O\left(\varepsilon^{2}\right) .
\end{aligned}
$$

The leading order term of the expression above is the solution of (1) obtained when holding the slow time $\left(t_{1}=\varepsilon t\right)$ constant. That is, to leading order, the carrying capacity may be considered to be varying slowly enough so that in comparison with the overall population change, it can be regarded as a constant. The second order term is an adjustment to the leading order, since the slow change in the carrying capacity does have an effect on the population as time passes. Note that substituting $t=0$ into (13) gives

$$
P(0, \varepsilon)=P_{0}+O\left(\varepsilon^{2}\right) ;
$$


that is, the expansion meets the required initial value, to the level considered. As $t \rightarrow \infty$, (13) gives

$$
P(t, \varepsilon) \rightarrow K(\varepsilon t)-\varepsilon r^{-1} K^{\prime}(\varepsilon t)+O\left(\varepsilon^{2}\right) ;
$$

that is, the expansion yields a limiting population close to, within $O(\varepsilon)$, but not equal to the carrying capacity. If $K^{\prime}(\varepsilon t)>0$; that is, the carrying capacity increases, this value is below $K(\varepsilon t)$; while if $K^{\prime}(\varepsilon t)<0$, it lies above. Note that, for a large class of population models, $K^{\prime}(\varepsilon t) \rightarrow 0$ as $t \rightarrow \infty$, and so the population does tend to $K(\varepsilon t)$; although the approach may be from above or below, as discussed above.

\section{The power law logistic equation}

The slowly varying logistic equation of Section 2 may be extended to a power law logistic model

$$
\frac{d P(t, \varepsilon)}{d t}=r P(t, \varepsilon)\left[1-\left(\frac{P(t, \varepsilon)}{K(\varepsilon t)}\right)^{s}\right], \quad P(0)=P_{0},
$$

where $s>0$. If the analysis of Section 3 is applied to this problem, we obtain a two term expansion for the population $P(t, \varepsilon)$

$$
\begin{aligned}
& P(t, \varepsilon)=\frac{K(\varepsilon t)}{\left(1+\left(\left(\frac{1}{P_{0}}\right)^{s}-\left(\frac{1}{K(0)}\right)^{s}\right) K(\varepsilon t) e^{-s r t}\right)^{1 / s}} \\
& -\varepsilon \frac{K^{\prime}(\varepsilon t)-K^{\prime}(0) K(0)^{-(s+1)} K(\varepsilon t)^{(s+1)} e^{-s r t}}{s r\left(1+\left(\left(\frac{1}{P(0)}\right)^{s}-\left(\frac{1}{K(0)}\right)^{s}\right) K(\varepsilon t) e^{-s r t}\right)^{1+1 / s}}+O\left(\varepsilon^{2}\right),
\end{aligned}
$$

analogous to (13). Note that when $s=1$, the above reduces to (13) as expected. Note also that in this case

$$
P(t, \varepsilon) \rightarrow K(\varepsilon t)-\varepsilon(r s)^{-1} K^{\prime}(\varepsilon t)+O\left(\varepsilon^{2}\right) ;
$$

so that the population displays limiting behaviour analogous to that of (13). 


\section{Discussion}

The expansions (13) and (15) provide explicit two-term approximations to the evolving populations arising from the logistic and power logistic models for arbitrary smooth slowly varying carrying capacities $K(\varepsilon t)$. These expansions are formal only, and the analysis necessary to validate them is well beyond the scope of this paper. Here, we demonstrate the plausibility of these results by applying (13) and (15) to specific examples of carrying capacity functions $K(\varepsilon t)$, that arise in modelling contexts, and comparing the results with those given by exact solutions (where these exist), or numerically constructed solutions.

In a number of applications $[1,4,5]$, the carrying capacity is itself generated as the solution of a logistic equation of the type (1) or (14), and may be represented in the general form

$$
K(\varepsilon t)=\frac{K^{*}}{\left(1+\left(\left(\frac{K^{*}}{K(0)}\right)^{\sigma}-1\right) e^{-\sigma \varepsilon t}\right)^{1 / \sigma}} .
$$

Here, the $s$ of (14) corresponds to the $\sigma$ above; and $\sigma=1$ is the more "usual" logistically generated carrying capacity. $K(0)$ is the initial value of $K(\varepsilon t)$, while $K^{*}$ is its limiting value as $t \rightarrow \infty$. When $\varepsilon$ is small (17) generates a slowly varying logistic function, which may be applied to (13) or (15) to yield an explicit two-term expansion for the evolving population $P(t, \varepsilon)$. Note: when $\sigma=s$ [1], the differential equation (14) incorporating $K(\varepsilon t)$ from (17) may be solved exactly subject to $P(0, \varepsilon)=P_{0}$, to give the solution

$$
P(t, \varepsilon)=\frac{K^{*}}{\left[1+\alpha e^{-\varepsilon s t}+(\beta-\alpha) e^{-r s t}\right]^{1 / s}},
$$

where

$$
\alpha=\frac{r}{r-\varepsilon}\left[\left(\frac{K^{*}}{K(0)}\right)^{s}-1\right], \quad \beta=\left(\frac{K(0)}{P(0)}\right)^{s}-1
$$




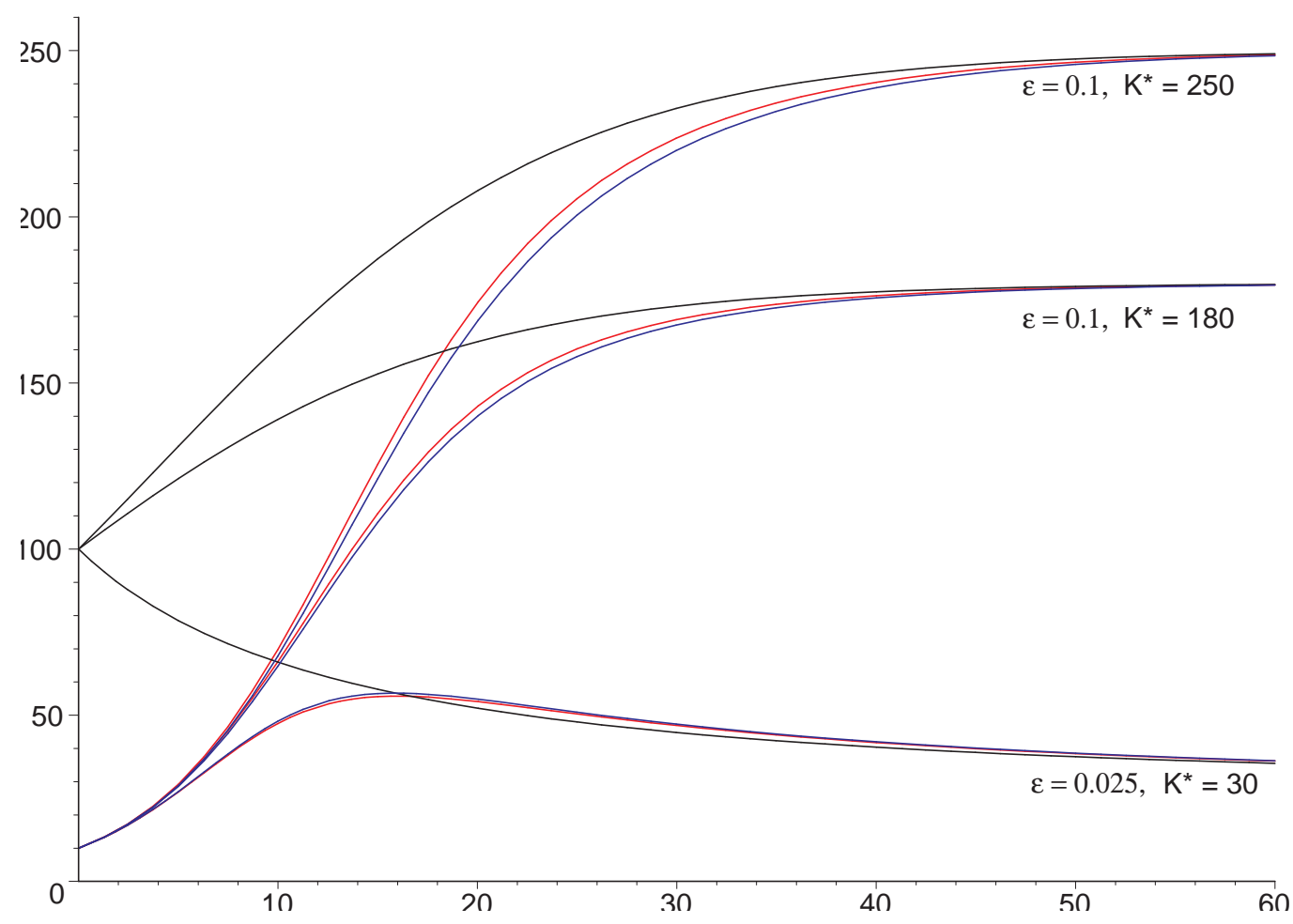

FiguRE 1: Approximation given by (13) compared with the exact solution ((18) with $\sigma=s=1)$. Black lines denote carrying capacity $K$, red lines denote the approximation and blue lines give the exact solution . 
Thus, when $\sigma=s$, this may be used as an indicator of the accuracy of the expansion described above.

Figure 1 makes such a comparison when $\sigma=s=1$, for a range of values of $\varepsilon$ and $K^{*}$. Clearly, the two term expansion approximates the exact solution very well over the range of time considered; and all display the desired overall behaviour. Note that both exact solution and approximation tend to $K$ from below when $K^{\prime}>0$ and above when $K^{\prime}<0$, as predicted.

When $\sigma \neq s$, the exact solution (18) is not available for comparison. In this case we compare expansions with the result of solving for the population $P$ using a numerical procedure (the analogue of an "exact solution"). Figure 2 compares the results provided by the leading term and the first two terms of (15) with a numerical solution when $\sigma=4$ and $s=2$. The second term of (15) provides a significant (and improving) correction to the leading term, with the full two-term expansion approximating the numerically generated curve very well indeed.

Often, $K$ shows a periodic variation about a positive mean value. This may be due to seasonal variations in the defining parameters, and since such variation is often on a much longer time scale, these variations take the nature of slow variation. Thus, typical periodic variation might be

$$
K(\varepsilon t)=K_{0}+\delta \sin (\varepsilon t),
$$

where $K_{0}$ and $\delta$ are positive constants, and $\varepsilon$ is small (and positive). This displays a slow oscillation of (large) period $2 \pi / \varepsilon$, amplitude $\delta$ and mean value $K_{0}$. (Note that for physical reality, we assume $\delta<K_{0}$, ensuring $K(\varepsilon t)>0$ for all $t \geq 0$.) For such $K$, the differential equation (1) (or (14)) may not be solved exactly. Banks [1] obtains an approximate representation of the solution; but here we use numerical techniques to obtain solutions for comparison with the results of using the two-term expansion (13), or (15), with (19).

Figure 3 shows the population variation (as approximated by (13)) resulting from a periodic $K$ of the form (19). This clearly shows the population 

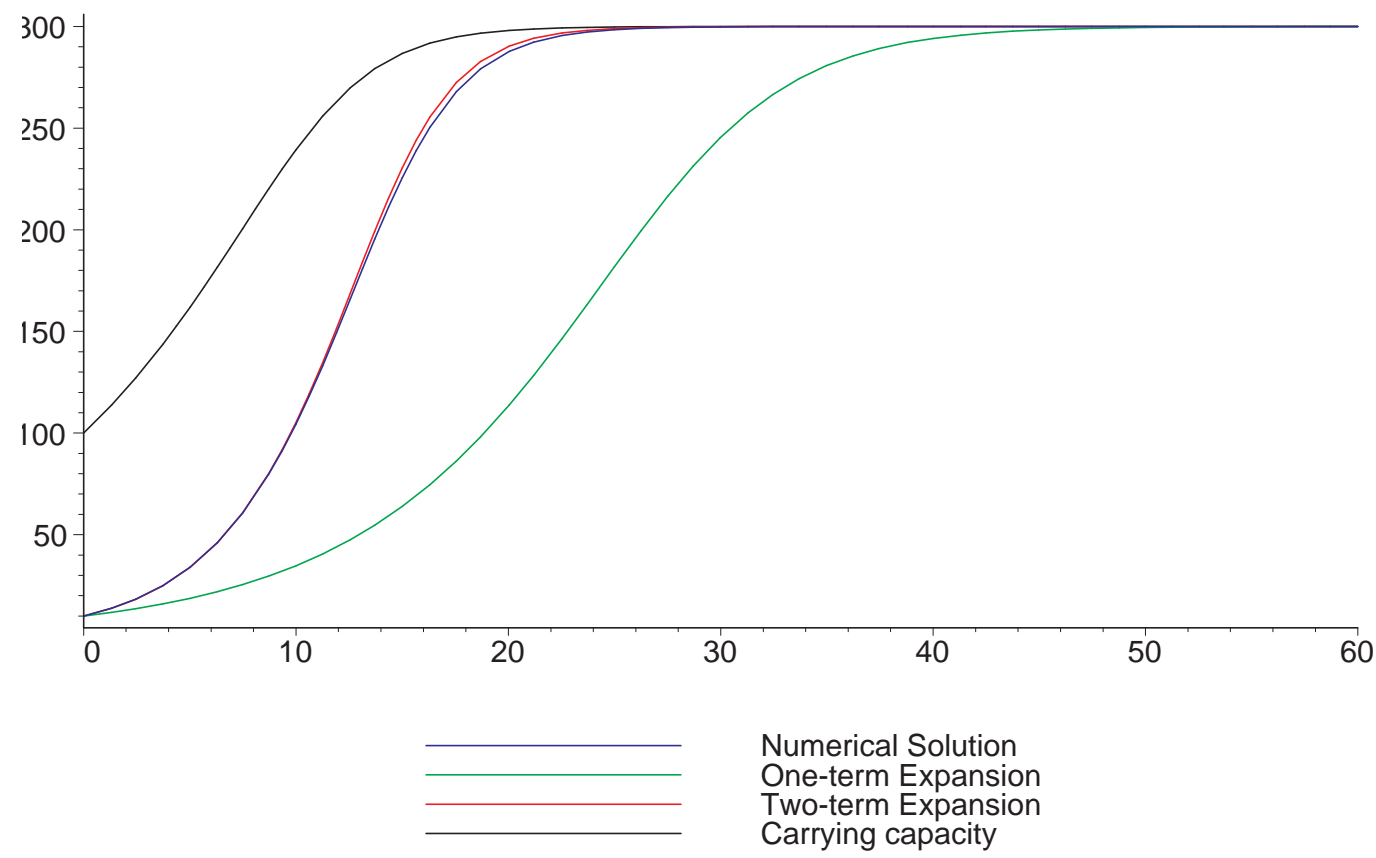

Figure 2: Comparison of the two term expansion (15) with numerical solution for power law logistically varying carrying capacity and population. Here $K(0)=100, P_{0}=10, r=0.25, K^{*}=300, \varepsilon=0.1, \sigma=4$ and $s=2$. 

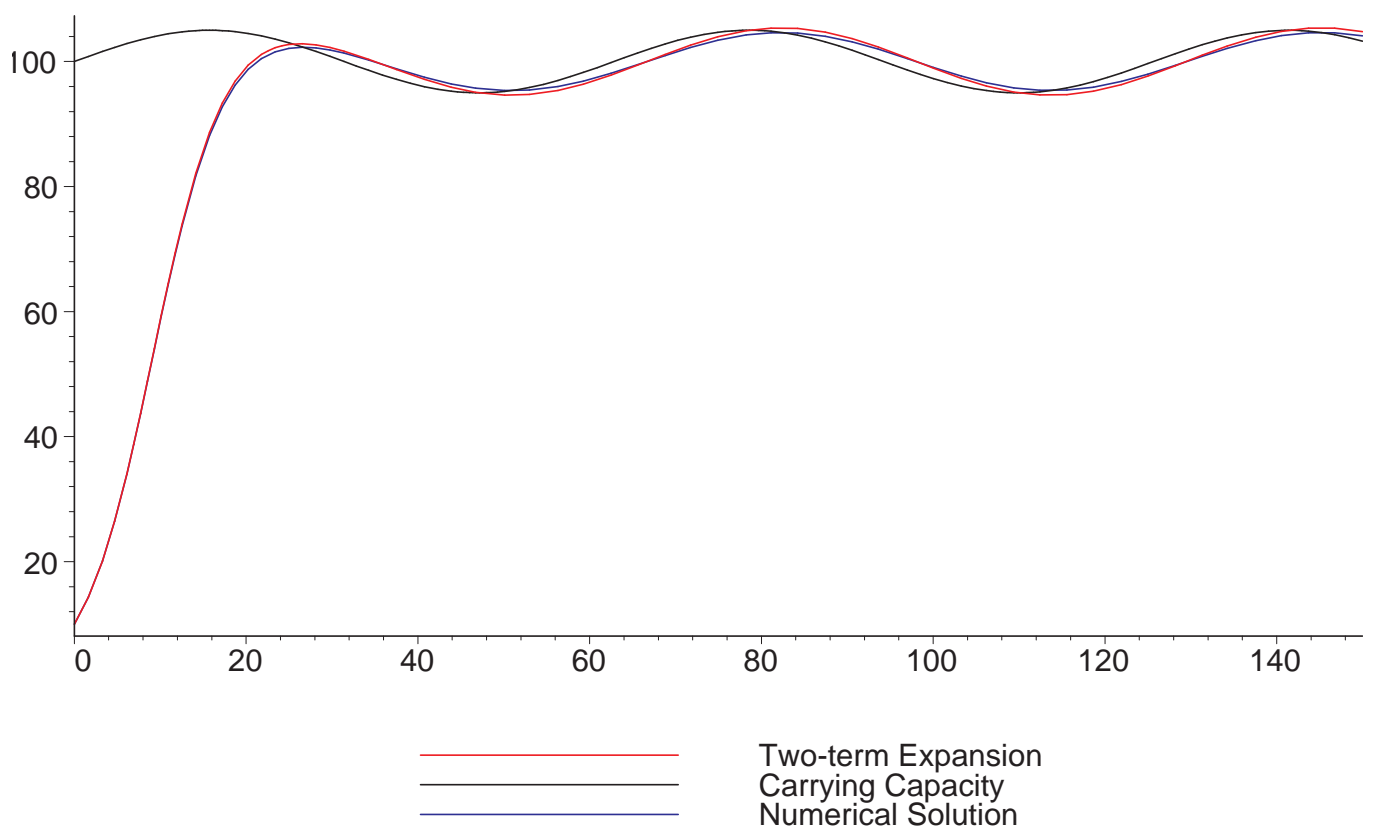

Figure 3: Population variation from a periodic $K$ for $K_{0}=100, P_{0}=5$, $r=0.25, \delta=10$ and $\varepsilon=0.1$. 
evolving to the point where it lies in a small neighbourhood of the carrying capacity, alternately lying above or below as the sign of $K^{\prime}$ changes. We also note the excellent agreement with the numerically generated solution.

The calculations demonstrate that the multi-timing method provides an explicit, accurate and convenient approximation to the exact solutions of single species population models when the carrying capacity displays slowly varying behaviour. These approximations are valid for a range of values of the parameters involved in the models considered, in contrast to the valuespecific results of numerical computations. Although we have only considered the logistic and power law logistic models here, it is apparent that the method is applicable to a range of such models - for example, the Gompertz model [2], or a logistic model with harvesting [6]. We are investigating this.

\section{References}

[1] Banks, R. B., Growth and Diffusion Phenomena : Mathematical Frameworks and Applications. Springer-Verlag, Berlin, Germany, 1994. C494, C500, C502

[2] Edelstein-Keshet, L., Mathematical Models in Biology, SIAM, USA, 2005. C505

[3] Holmes, M. H., Introduction to Perturbation Methods Springer, New York, 1995. C494

[4] Meyer, P., Bi-logistic growth, Technological Forecasting and Social Change 47, 89-102, 1994. doi:10.1016/0040-1625(94)90042-6 C493, C500

[5] Meyer, P. S., Ausubel, J. H., Carrying capacity: a model with logistically varying limits, Technological Forecasting and Social Change 61(3): 209-214, 1999. doi:10.1016/S0040-1625(99)00022-0 C493, C500 
[6] Murray, J. D., Mathematical Biology I. An Introduction 3rd Ed, Springer-Verlag, Berlin, 2002. C493, C505

[7] Nayfeh, A. H., Perturbation Methods John Wiley \& Sons, 1973. C494 